Dermatology

\section{Hyperpigmented rugosity of skin associated with adenocarcinoma of the caecum}

\section{A C Inamadar, A Palit, B R Yelikar}

A 52 year old male office worker presented with localised areas of dark, rugose skin over the face, dorsa of the hands and feet of 15 days' duration. The lesions were asymptomatic but rapidly progressive causing concern to the patient. In addition, he had weight loss (>10 kg), anorexia, and profound weakness for the past month. He was non-smoker and non-alcoholic. He had been suffering from bleeding piles and anal fissures for the past 15 years. There was no history of fever, cough, respiratory distress, change of bowel habits, or difficulties in micturition.

On general physical examination there was moderate pallor. Systemic examinations were normal. Local cutaneous examination showed hyperpigmented, velvety, rugosities distributed over the forehead, bridge of the nose, and malar areas of the face (fig 1). Similar lesions were present over the knuckles and anterior aspect of the ankles (fig 2). Palms, soles, lips, and oral mucosa were normal. The patient was investigated for the aetiological

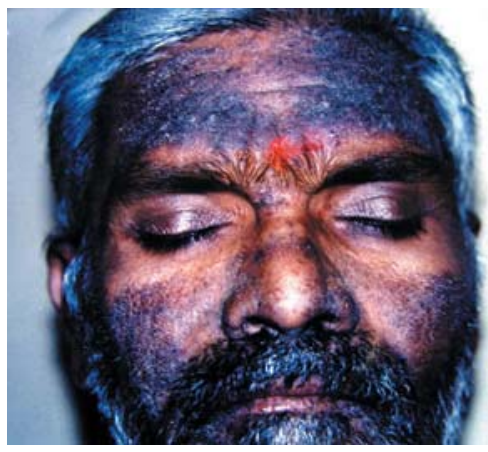

Figure 1 Hyperpigmented velvety, plaque lesions over face. confirmation of recent onset weight loss. Complete haemogram showed a haemoglobin of $8 \mathrm{~g} \%$, raised erythrocyte sedimentation rate ( $80 \mathrm{~mm}$ in lst hour), and a hypochromic, microcytic peripheral blood smear. Routine examination of urine, stool, and chest radiograph were normal. Enzyme linked immunosorbent assay for HIV was negative. Rectal examination showed a normal prostate. Upper gastrointestinal endoscopy was normal. Colonoscopy showed a caecal bulge with a small, friable ulcer in anterior wall. An endoscopic biopsy sample comprising the entire ulcerated area, measuring $0.4 \mathrm{~cm}$, was taken. Histopathological examination showed dysplastic, large, irregular glandular epithelium with hyperchromatic nuclei, prominent nucleoli, and mitotic figures. Lamina propria and muscularis mucosae were infiltrated with pleomorphic cells. The features were consistent with moderately differentiated, infiltrative adenocarcinoma

Excision of caecum including adjacent terminal ileum, ascending colon, and lymph nodes was performed followed by end to end anastomosis. The excised specimen showed unremarkable

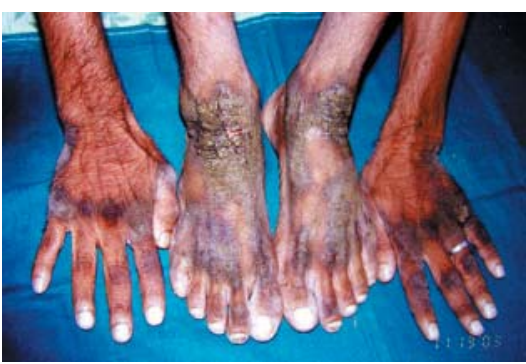

Figure 2 Verrucous plaques over knuckles and front of the ankles.

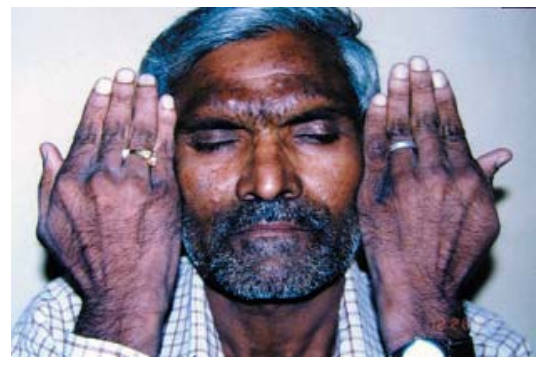

Figure 3 Regression of the lesions two weeks after surgery.

serosa and mucosa without any evidence of tumour. Discrete, multiple lymph nodes of varying size were identified. There was no evidence of malignancy on serial sections of the specimen of the caecum. Section from all the lymph nodes showed features of reactive lymphadenitis.

During the postoperative period, there was remarkable regression of the skin lesions (fig 3). On subsequent follow up, there was no symptom pertaining to the recurrence of the colonic lesion. Complete clearance of skin lesions was seen four weeks later. The patient has been kept under regular surveillance for evidence of recurrence or metastasis of the colonic lesion and relapse of the skin lesions.

\section{QUESTIONS}

(1) What is the terminology used for this dermatosis?

(2) What are the variants of this disorder?

(3) How does the condition differ clinically when associated with malignancy?

Postgrad Med J 2005;81:271

doi: $10.1136 /$ pgmi.2004.020933

\section{Authors' affiliations}

A C Inamadar, A Palit, Department of Dermatology, BLDEA's SBMP Medical College, Hospital and Research Centre, Bijapur, Karnataka, India

B R Yelikar, Department of Pathology, BLDEA's SBMP Medical College, Hospital and Research Centre

Correspondence to: Professor A C Inamadar, Department of Dermatology, BLDEA's SBMP Medical College, Hospital and Research Centre, Bijapur 586103, Karnataka, India; Aparunal@rediffmail.com

Submitted 23 February 2004

Accepted 26 May 2004 